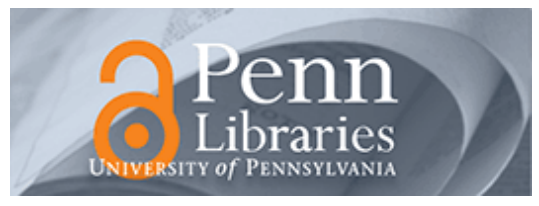

University of Pennsylvania

ScholarlyCommons

Management Papers

Wharton Faculty Research

$7-2002$

\title{
Misperceiving Interactions Among Complements and Substitutes: Organizational Consequences
}

Nicolaj Siggelkow

University of Pennsylvania

Follow this and additional works at: https://repository.upenn.edu/mgmt_papers

Part of the Business Administration, Management, and Operations Commons

\section{Recommended Citation}

Siggelkow, N. (2002). Misperceiving Interactions Among Complements and Substitutes: Organizational Consequences. Management Science, 48 (7), 900-916. http://dx.doi.org/10.1287/mnsc.48.7.900.2820

This paper is posted at ScholarlyCommons. https://repository.upenn.edu/mgmt_papers/132

For more information, please contact repository@pobox.upenn.edu. 


\title{
Misperceiving Interactions Among Complements and Substitutes: Organizational Consequences
}

\author{
Abstract \\ Systems composed of activity choices that interact in nonsimple ways can allow firms to create and \\ sustain a competitive advantage. However, in complex systems, decision makers may not always have a \\ precise understanding of the exact strength of the interaction between activities. Likewise, incentive and \\ accounting systems may lead decision makers to ignore or misperceive interactions. This paper studies \\ formally the consequences of misperceiving interaction effects between activity choices. Our results \\ suggest that misperceptions with respect to complements are more costly than with respect to \\ substitutes. As a result, firms should optimally invest more to gather information about interactions \\ among complementary activities-e.g., concerning network effects-than about interactions among \\ substitute activities. Similarly, the use of division-based incentive schemes appears to be more advisable \\ for divisions whose products are substitutes than for divisions that produce complements. It is further \\ shown that system fragility is not necessarily positively correlated with the strength ofthe interaction \\ between choices. While systems of complements become increasingly fragile as the strength of \\ interaction increases, systems of substitutes can become increasingly stable.
}

\section{Keywords}

interactions, systems of activities, complementarities, misperceptions, substitutes, organizational design, supermodularity, fragility, complexity, division-based incentives

\section{Disciplines}

Business Administration, Management, and Operations 


\title{
Misperceiving Interactions: Organizational Consequences
}

\author{
Nicolaj Siggelkow \\ Management Department \\ Wharton School \\ 2017 Steinberg Hall - Dietrich Hall \\ University of Pennsylvania \\ Philadelphia, PA 19104 \\ siggelkow@wharton.upenn.edu \\ tel: (215) 573-7137 \\ fax: (215) 898-0401
}

Draft date: March 28, 2001

I would like to thank Rebecca Henderson, Bruce Kogut, Daniel Levinthal, Nicola Persico, Madhav Rajan, Jan Rivkin, Scott Schaefer, Scott Stern, Christian Terwiesch, Keith Weigelt, and seminar participants at the University of Pennsylvania and at Stanford University for helpful comments. All remaining errors are mine. Financial support by the Reginald H. Jones Center for Management Strategy, Policy and Organization is gratefully acknowledged. 


\title{
Misperceiving Interactions: Organizational Consequences
}

\begin{abstract}
:
Systems composed of activity choices that interact in non-simple ways can allow firms to create and sustain a competitive advantage. However, in complex systems, decision makers may not always have a precise understanding about the exact strength of the interaction between activities. This paper studies formally the consequences of misperceiving interaction effects between activity choices. Our results suggest that misperceptions with respect to complements are more costly than with respect to substitutes. It is also shown that system fragility is not necessarily positively correlated with the strength of the interaction between choices. While systems of complements become increasingly fragile as the strength of interaction increases, systems of substitutes can become increasingly stable. By pointing towards those types of activities for which increased information about their interactions tends to yield high benefits, the model offers insights with respect to organizational design.
\end{abstract}




\section{Introduction}

In recent years, the topic of interaction among activity choices of firms has received a burst of attention in the organization, economics, and management literatures (e.g., Levinthal 1997; Milgrom and Roberts 1990a; Porter 1996). In particular, it has been argued that systems of tightly interconnected activities play an important role in creating and sustaining a competitive advantage (Milgrom and Roberts 1995; Rivkin 2000). In contrast to these positive views of tight interconnectedness, another stream of literature has analyzed the potential downside of tight linkages, in particular in the context of changing environmental conditions (Levinthal 1997; Siggelkow forthcoming). In this paper, we add a new dimension to the analysis of tightly coupled systems. In complex systems, boundedly rational decision makers (Simon 1957) are not likely to have a precise understanding of all interaction effects among the decisions they are engaged in. Decision makers may ignore interaction effects, may over- or underestimate them, or have some uncertainty around their true value. We set out to study for which types of interactions these misperceptions are likely to generate large performance declines. The answer to this question points to those situations in which a reduction of misperceptions of interaction effects is particularly valuable. Since information about interaction effects—and, hence, misperceptions—are influenced by a variety of organizational factors (e.g., job assignments, task partitions, organizational design, boundaries of firms), the results have potential implications for a broad range of issues. Thus, at a general level, this paper adds to the organizational "better design approach," which centers on the idea that the appropriate design of organizations can mitigate the impact of information distortions (Baligh, Burton, and Obel 1996; Burton and Obel 1984; Carley 1992; Carley and Lin 1997; Malone 1987).

While most informal treatments of interaction effects have discussed "interactions" generically, this paper focuses on the distinction between complementary interactions and interactions of substitutes. Two activities are said to interact as substitutes, if the marginal benefit of each activity decreases in the level of the other activity. Two activities interact as complements, if the marginal benefit of each activity increases in the level of the other activity. The results of our analyses suggest that complementary interactions behave quite differently from substitute interactions. In particular, misperceptions of 
complementary interactions tend to be more detrimental than misperceptions of substitute interactions. Moreover, systems of complements tend to become more fragile as the strength of interaction increases, while systems of substitutes can become more robust as interactions strengthen. In an extension of the basic model, the paper addresses the further question whether it is preferable for managers to have the same mistaken belief about interactions or to have independent beliefs.

\section{Misperceptions in systems of interconnected choices}

Systems of interconnected choices exist at various levels of analysis and organizational complexity. For instance, a product design project is a within-firm system of interconnected choices at a very finegrained level. In this case, each design parameter corresponds to a choice. At a higher organizational level, the (Japanese) lean manufacturing methods have been shown to consist of systems of interconnected practices (Jaikumar 1986; MacDuffie 1995; Milgrom and Roberts 1990a; Nemetz and Fry 1988; Womack, Jones, and Roos 1990). At an even broader level, a firm as a whole can be seen as a system or configuration of interconnected choices: choices with respect to its product portfolio, activities, resources, policies etc. (Drazin and Van de Ven 1985; Milgrom and Roberts 1995; Miller and Friesen 1984; Porter 1996; Siggelkow forthcoming). Systems of interconnected choices can also cross organizational boundaries. At the level of an individual product, when sub-components are outsourced to suppliers, the (product-) system spans several organizations (Garud and Kumaraswamy 1995). Lastly, on a larger scale, alliances between firms have many characteristics of interconnected systems, with alliance partners decomposing tasks and finding ways to manage the interdependencies among these tasks (Gulati and Singh 1998).

Misperceptions about interactions can arise in all of the systems described above. For instance, in the context of product development, Eppinger (1994) notes that "interactions [among tasks] are often poorly understood and are rarely known in advance" (p. 1). This lack of knowledge raises problems for concurrent engineering, which has become a common mode of product development (Clark and Fujimoto 1991; Ulrich and Krishnan 2001). Designers may, for instance, believe that two interdependent tasks are 
independent and as a result allocate them to two different modules that are worked on concurrently. Since the interaction between the two tasks is not taken into account, the resulting design is likely to be suboptimal and may require additional, costly design iterations. Thus, one source of misperception is simple lack of knowledge or foresight about interactions.

In the following, we elaborate on two other, common sources of misperceptions. First, since decision problems with many interactive elements are very difficult to solve (Rivkin 2000), many firms use simplification strategies. These simplifications can lead to interactions' being ignored or distorted. Examples of such simplifications include common R\&D project selection rules (Steele 1988), profitcenter incentive systems (Dearden 1987), linear assumptions in cost accounting systems (Banker and Atkinson 1996), and traditional fixed-cost allocation rules (Cooper and Kaplan 1988). A second source of misperceptions are outdated managerial mental models about interaction effects. While some interactions are ignored due to simplification strategies, other interactions are studied and internalized by managers and organizations in the form of heuristics and organizational routines (Nelson and Winter 1982). Managerial mental models that reflect interactions are, however, slow to adapt (Hambrick and Mason 1984; Kiesler and Sproull 1982; Murmann and Tushman 1997). Thus, if underlying interaction pattern change, mental models can become outdated, leading to interactions that are ignored or misperceived (Henderson and Clark 1990). Several examples of such misperceptions are discussed below.

Misperceptions caused by simplifications. R\&D project selection provides an example in which widely used decision rules that simplify a complex problem with many interaction effects lead to ignored interactions. Benefit measurement methods are the most widely used practices for $R \& D$ project selection (Steele 1988; Hall and Nauda 1990). Projects are ranked according to a number of weighted decision criteria and the highest ranking projects are picked (within an overall budget constraint). Commonly, projects are ranked on a single criterion such as net present value and projects with the highest values are selected. The drawback of this method is that interactions among projects are ignored. The value of investing in one project may be influenced by the amount that is invested in a different project. For 
instance, the value of developing a new transmission system, which increases fuel efficiency but increases noise, is enhanced by a project that is geared towards reducing noise stemming from the exhaust system. At the same time, if overall value of increasing fuel efficiency has decreasing returns, a project that increases engine fuel efficiency would decrease the marginal benefit of developing a new transmission system that increases fuel efficiency as well. Thus, projects can interact as substitutes or as complements. While a considerable academic literature has developed methodologies that can take interactions in $R \& D$ project selection into account, these quantitative project-selection models continue to be ignored in practice (Steele 1988; Burnett, Silverman, and Monetta 1993).

In general, interactions are misperceived (or ignored) when externalities of actions are not fully taken into account. For instance, many firms have created "profit centers" inside the corporation (Reece and Cool 1978) and have tied managers' compensation to their profit centers' performance (Dearden 1987). On the one hand, this simplifies a manager's decisions process, since consequences of actions beyond her division can be ignored. On the other hand, since externalities are ignored, actions might be taken that are suboptimal from the firm's overall perspective. Consider an investment decision by a manager of a profit center. If the manager's compensation is entirely linked to her division performance, she will choose an investment level that maximizes her division's performance. If the investment in her division affects other divisions as well, she would not take this interaction into account. Thus, her investment decision would appear as if she ignored the interaction. (If her compensation is partially linked to overall firm performance, she would take the interaction into account, but only partially.) Fundamentally, the investment distortion is caused by the manager's using her division's marginal benefit rather than the firm's overall marginal benefit in making her investment decision.

Similar problems can arise from cost accounting systems that decompose costs linearly-a feature shared by most cost accounting systems (Banker and Atkinson 1996). For instance, the difference between the expected and the actual manufacturing cost of a component is commonly decomposed into a "price variance" (the price of the inputs was different than expected) and an "efficiency variance" (the amount of inputs used was different). These variances, in turn, are used to hold different managers 
accountable. The purchasing manager's compensation is linked to the price variance while the operation's manager's compensation is linked to the efficiency variance. The information generated by the cost accounting system, and the incentive system that is linked to it, can lead to inefficiencies in behavior when the two variances interact, which is likely to be the case. For instance, buying material of lesser quality will help the price variance, yet hurt the efficiency variance. Even if individual managers wanted to maximize total firm performance, the firm-optimal tradeoffs, which take interdependencies into account, are difficult for the managers to achieve given the information provided to them by the cost accounting system.

The above examples illustrate how accounting systems and the accompanying incentive systems may cause interactions to be ignored. Cost accounting systems, with embedded simplifying assumptions, may also introduce interactions, where no interactions exist. Consider the allocation of overhead costs. In many traditional cost accounting systems, set-up costs are treated as overhead, and total overhead costs are distributed according to machine run-time or direct labor hours (Cooper and Kaplan 1988). Consequently, a large amount of total overhead costs would be allocated to a high-volume product, even though set-up costs might be fixed, i.e., independent of production volume. Moreover, as the output of this product increases, the fixed costs allocated to another product decreases. As a result, the total cost of the second product is a function of the production volume of the first product. Thus, the cost accounting system introduces an interaction between the costs of these two products. However, if set-up costs are fixed and allocated to their correct sources, no such interdependency would truly exist.

The consequences of misallocated fixed costs can be serious and have led many firms to take remedial actions, e.g., to install activity-based costing systems that ascribe each (fixed) cost to its source (Cooper and Kaplan 1988). More generally, the problem of ignoring interaction effects between decisions has received serious attention in many firms (providing indirect evidence for the importance that firms attach to these problems), as can be seen in firms' attempts to create measurement systems that take more interdependencies into account. For instance, the "balanced scorecard" (Kaplan and Norton 1992) 
requires managers to take into account the effects of their decisions not only on short-term financial performance, but also on customer perceptions and the firm's long-term innovative capabilities.

Misperceptions caused by outdated mental models. As a second source for misperceptions we consider outdated managerial mental models and their associated routines (Nelson and Winter 1982). When interaction effects between activities change, routines and heuristics (e.g., "if spending on design technology increases by $10 \%$, investments on manufacturing technology should be increased by $10 \%$ as well") may not adapt immediately. Managers using the old routines would then behave as if they "misperceived" the true interaction effect. In the context of technological innovation, Henderson and Clark (1990) and Henderson (1993) document how firms with deeply-ingrained organizational routines have a difficult time responding to shifts in knowledge that relate to new ways in which parts of a system (a product or a production process) interact with each other, i.e., to changes in interaction effects. Similar findings have been reported by studies that document the struggles encountered by U.S. firms that adopted lean manufacturing practices. In their attempts to replicate lean manufacturing, many managers failed to recognize that the value of individual practices was greatly affected by the presence of other practices (Hayes and Jaikumar 1988). For instance, the value of flexible manufacturing systems was greatly enhanced by concurrent investment in worker education and a broadening of the product line, yet originally many U.S. firms were apparently not aware of these interaction effects and changed their existing system only in a detrimental, incremental fashion (Jaikumar 1986).

In the realm of manufacturing, outdated mental models can arise because the lean manufacturing system involves changes in many activity interactions. As MacDuffie (1995) notes, “A flexible production plant reduces inventory levels and other 'buffers,' increasing interdependence" (p. 198). The elimination of many buffers in the production process has, in particular, increased the number of complementary relationships. For instance, if no build-up of work-in-progress inventory is allowed, i.e., if output is only generated when all sequential production units operate, the marginal benefit of increasing 
up-time for one unit is increasing in the up-time of all other production units. In other words, the investments of increasing up-time of the various units have become complementary (Kremer 1993).

The increase in the number of interactions among business processes does not seem to be confined to manufacturing. Analyzing the results of an extensive survey of large and medium-sized companies across Europe, Whittington et al. (1999) find "significant increases in horizontal interactions" (p. 590). In particular, 74\% of companies reported that the importance of horizontal linkages such as sharing of R\&D knowledge across units, joint purchasing, common distribution channels, and shared marketing information had increased between 1992 and 1996. Thus, if manager's mental maps of cause-and-effect relationships do not adjust as rapidly as the true underlying relationships change, misperceptions of interactions can arise.

A detailed example of misperceptions of interaction effects due to outdated managerial mental models is provided by Siggelkow (forthcoming). In the early 1990s, Liz Claiborne, a fashion apparel manufacturer, attempted to start a reordering program for its retail customers. As shown by Siggelkow (forthcoming), Liz Claiborne had constructed an intricate system of interconnected choices that had allowed Liz Claiborne to create great shareholder value over the 1980s. When starting the reordering program, Liz Claiborne's management changed only one element of this system: it allowed department store buyers to reorder individual items and promised to deliver within two weeks. However, the previous choice of "no-reordering" interacted with many other choices of Liz Claiborne, e.g., its choice of contract manufacturers in the Far East, which did not allow production-to-order, and its small investments in information technology. Existing management, however, ignored these interactions and attempted to implement the reordering system in a piecemeal fashion, leading to a large inventory build-up and consequent write-offs that had a large impact on Liz Claiborne's profitability. With no expertise concerning the new reordering system, Liz Claiborne's management either did not know about these interactions, or greatly underestimated the magnitude of these interactions. Moreover, Liz Claiborne's accounting system was not set up to generate information about inventory costs. Hence, management did not receive timely information about the costs of their failed attempt to install the reordering system. 
The above examples illustrate a number of sources of misperceptions about interaction effects. Once misperceptions exist, how likely are they to persist, though? Put differently, how easy or difficult is learning about interaction effects? Experimental data suggests that learning about interaction effects is very difficult. While the models of Sterman (1989a; 1989b) and Diehl and Sterman (1995) are dynamic in nature (focusing on dynamic feedback effects rather than on static interactions), and thus, not fully comparable to the model developed in this paper, the evidence is nonetheless intriguing. Subjects are found to misperceive both positive and negative feedback effects (Diehl and Sterman 1995). Moreover, the majority of the underperformance (relative to optimal performance) arises from subjects employing a poor model, i.e., from misperceptions, rather than from employing the right model inconsistently (Diehl and Sterman 1995; see also Bowman 1963). As Sterman (1994) concludes "learning about complex systems when you also live in them is difficult" (p.292). Thus, misperceptions, once formed, are not easily corrected.

\section{Related literature}

To show the relationship between the model developed in this paper and existing work, it is helpful to provide a short preview of the model. In the model developed below, one or two managers choose the levels of two interconnected activities, $A$ and $B$, in order to maximize an objective function $V=f(A, B, \alpha)$, where $\alpha$ is an exogenous parameter determining the interaction. If $\alpha$ is positive, $A$ and $B$ are complements; if $\alpha$ is negative, $A$ and $B$ are substitutes. Managers may misperceive the true interaction parameter $\alpha$ to be $z$. For instance, if $z=0$ while $\alpha \neq 0$, managers are said to ignore the interaction. The model studies for which values of $\alpha$ misperceptions are particularly detrimental (as compared to the case of no misperception).

The discussion of related work is divided into four sections. First, for positive values of $\alpha$ the function $f$ is supermodular in the choice variables (for an exact definition of supermodularity, see below). Since supermodularity plays a central role in the recent literature on monotone comparative statics, this 
literature is discussed first. Second, a stream of work has analyzed the value of information under conditions of uncertainty. This work is directly related since the value of information is the converse of the performance decline that is studied in this paper. Third, we provide a short description of formal work that has employed a similar functional set-up as the one analyzed in this paper. Fourth, we conclude with a brief discussion of related work in the organization literature.

Monotone comparative statics. Consider a function $f(x, \theta)$, where $\boldsymbol{x}$ is a vector of choice variables and $\theta$ is a parameter. The work on monotone comparative statics is concerned with the following question: If a single decision maker maximizes $f$ (given a constraint set for $\boldsymbol{x}$ ), under which conditions on $f$ is the optimal choice $\boldsymbol{x} *(\theta)$ nondecreasing in $\theta$ ? Put differently, under what conditions does an increase in an exogenous parameter $\theta$ lead (weakly) to an increase in all optimized choice variables $\boldsymbol{x}$ ? Topkis (1978) shows that this monotone comparative static result holds if $f$ is supermodular in $\boldsymbol{x}$ and $f$ has increasing differences in $(x ; \theta)$. (For formal definitions of italicized terms, see Appendix 1.) For a general definition of supermodularity that does not require differentiability of $f$ see also Appendix 1. For the differentiable case, which is sufficient for the model we investigate, Topkis (1978) proves that $f$ is supermodular if $\frac{\partial f^{2}}{\partial x_{i} \partial x_{j}} \geq 0$ for all $x_{i} \neq x_{j}$

Milgrom and Shannon (1994) extend the work of Topkis (1978) and show that the monotone comparative statics result holds for slightly weaker conditions on $f$. In particular, if $f$ is quasisupermodular in $\boldsymbol{x}$ and satisfies the single-crossing property in $(\boldsymbol{x} ; \theta)$, then there exists a $\boldsymbol{x} *(\theta)$ that is nondecreasing in $\theta$. Note, $x *(\theta)$ may not be unique. Thus, there may be a selection from the set of maximizers that is not nondecreasing. In further work, Shannon (1995) explores the stronger conditions under which any selection from the set of maximizers is nondecreasing in $\theta$, and the weaker conditions for which at least one selection from the set of maximizers exists that is nondecreasing in $\theta$. While the results in the preceding papers guarantee nondecreasing maximizers, Edlin and Shannon (1998) explore the conditions under which the maximizers are strictly increasing in $\theta$. Friedman and Johnson (1997) 
extend the comparative static results to a dynamic setting. Here, the payoff function for each period is taken to be $f\left(\boldsymbol{x}_{t}, \boldsymbol{x}_{t-1}, s_{t}, \theta\right)$, where $\boldsymbol{x}_{t}$ is the choice variable in period $t, s_{t}$ is an exogenous stationary, Markovian random process with positive persistence of uncertainty (e.g., a market price), and $\theta$ an exogenous parameter. Friedman and Johnson (1997) show that if $f$ is supermodular in the actions and has nondecreasing differences in actions and the exogenous parameters, the optimal decision rule $\boldsymbol{x}^{*}\left(\boldsymbol{x}_{t-1}, s_{t}, \boldsymbol{\theta}\right)$ is nondecreasing in $s_{t}$ and $\theta$. Lastly, Athey (forthcoming-a) explores more general stochastic problems in which the payoff to actions is affected by the realization of a vector of random variables, whose joint density function in turn is influenced by an exogenous parameter. Thus, $f(\boldsymbol{x}, \theta)=\int u(\boldsymbol{x}, \boldsymbol{s}) g(\boldsymbol{s}, \boldsymbol{\theta}) d s$ where $u$ is the agent's payoff function, $\boldsymbol{x}$ represents a choice vector, $\theta$ is an exogenous parameter, and $g$ is the joint probability density function over a vector of random variables $s$. Athey establishes necessary and sufficient conditions for the agent's optimal choice $\boldsymbol{x}^{*}(\theta)$ to be nondecreasing in $\theta$. In particular, she shows that $\boldsymbol{x}^{*}(\theta)$ is nondecreasing in $\theta$ for all payoff functions $u$ that are log-supermodular if and only if $g$ is log-supermodular. ${ }^{1}$

Comparing signals and value of information. A second stream of literature has studied the value of signals that carry information about unknown parameters. Assume a decision maker's payoff $f$ depends both on her real-valued action $x$ and the state of the world $\theta$, i.e., $f(x, \theta)$. The agent considers $\theta$ to be a realization of a random variable $\Theta$. Moreover, $\theta$ is not observable. However, the decision maker observes the realization of a signal $S$, which is also a random variable, and which carries some information about the true realization of $\theta$. Consider two signals $S$ and $S^{\prime}$. Blackwell $(1951 ; 1953)$ shows the conditions on $S$ and $S^{\prime}$ so that $S^{\prime}$ is more valuable for the decision maker (in an expected sense) for any decision problem. These conditions (statistical sufficiency) turn out to be very restrictive, leading various authors to restrict the set of decision problems under consideration in order to derive broader conclusions. For instance, Jewitt (1989) restricts $f$ to be concave (making $\theta$ a measure of risk aversion). A decision maker has to choose between projects with stochastic payoffs and choose the project that maximizes expected utility. 
Let $F_{1}$ and $F_{2}$ be the distribution functions of payoffs from two projects. Jewitt (1989) studies the conditions on $F_{1}$ and $F_{2}$ such that if a decision maker prefers $F_{1}$ to $F_{2}$, then so will all decision makers who are more risk averse.

Lehman (1988) starts a different line of inquiry by restricting the set of decision problems to monotone decision problems. A decision problem is monotone if observing a higher signal realization induces a higher action. Athey and Levin (2000) continue the study of monotone decision problems by considering general sets of payoff functions that are alike in how the incremental returns to higher actions change with $\theta$ (e.g., the set of supermodular payoff functions, for which the returns to increasing the action are nondecreasing in $\theta$.) Athey and Levin (2000) are concerned with two problems. First, following Blackwell $(1951 ; 1953)$ and Lehman (1988), they derive conditions under which for all agents within a family of decision makers observing a signal $S^{\prime}$ is more valuable than observing $S$. Second, they provide conditions under which the incentives of two agents to acquire better information can be ranked. For instance, they show that a monopolist will acquire less information on (uncertain) marginal cost than a social planner would. Encompassing a wider range of payoff functions, Athey and Levin's work also extends the work of Persico (2000) who similarly studies for what conditions on $f$ information about $\theta$ is more valuable. It is important to note that both the frameworks of Athey and Levin (2000) and Persico (2000) consider only a single choice $x$ (e.g., a bidding price in the auction model of Persico). As a result $f$ is (trivially) supermodular in the choice $x$.

The model studied in this paper has also the form of decision makers' choosing optimal choices $\boldsymbol{x}^{*}$ to maximize a function $f(x, \alpha)$. Similar to the work described in the last paragraph, it is assumed that the decision makers do not observe the exogenous parameter $\alpha$ but some signal $z$. The signal $z$ is modeled to be either fixed or random. The key difference to the literature discussed above is that in our model the value of $\alpha$ determines whether $f$ is supermodular in $\boldsymbol{x}$ or not. (For this reason, we refer to this parameter as $\alpha$ rather than as $\theta$.) While the questions we raise are similar to the ones studied by Athey and Levin (2000) and Persico (2000) — namely the value of information about $\alpha$ given the properties of $f$ - two 
significant differences exist. First, and central to our model, $f$ is not always supermodular in $\boldsymbol{x}$. We break supermodularity by considering an $\boldsymbol{x}$ that is more than one-dimensional and allowing $\alpha$ to affect the interaction between the components of $\boldsymbol{x}$. Hence, this model is not a special case of the more general setups of Athey and Levin (2000) or Persico (2000). Second, rather than having a single decision maker, we allow the decisions on the various components on $\boldsymbol{x}$ to be made by different decision makers.

Work with similar functional assumption. We believe that the study of interaction effects beyond supermodularity is important because, as described by Porter and Siggelkow (2001), activities in firms interact in a variety of ways that do not fulfill the supermodularity conditions. By going beyond supermodularity, we pay, however, a price in this paper in terms of the generality of the approach. To take a first step towards studying the effects of misperceptions in this broader context, we employ a specific functional form by assuming $f$ to be quadratic. Schaefer (1999), in studying the optimal design partitions when components interact in a supermodular manner, takes a similar approach. In order to extend a finding by Milgrom and Roberts (to be discussed in more detail in Section V), he uses a quadratic functional form rather than a general supermodular function.

Structurally, the closest approach to the one taken in this paper has been employed by Crémer (1990; 1993). Crémer studies the relative value of homogenous vs. heterogeneous information within the context of a quadratic production function (see Section V). While in our set-up misperceptions occur with respect to the quadratic interaction term, in Crémer's model the misperception occurs with respect to the linear terms. More generally, the quadratic function has a long tradition in the theory of teams (Groves and Radner 1972; Marschak and Radner 1972; Radner 1972) and in models of organizational hierarchies (Geanakoplos and Milgrom 1991). Similarly, in a model of worker-matching, Kremer (1993) uses a related multiplicative production function of the form $f(\boldsymbol{x})=\theta x_{1} x_{2} x_{3} \ldots x_{n}$ with $\theta>0$, which describes a production process with sequential tasks, each of which is completed successfully with probability $x_{i}$. (See also footnote 3). If worker skill is measured by $x_{i}$, Kremer (1993) shows that workers with the same skill level will be matched together in equilibrium and that small differences in worker skill create large 
differences in productivity and wages. Kremer (1993) further shows that these results generalize to symmetric production functions with positive cross-derivatives in worker skill, i.e., for continuous functions that are supermodular in $\boldsymbol{x}$. If cross-partials are negative, Kremer and Maskin (1996) show that the highest and lowest skill workers will be grouped together in equilibrium.

Previous work in the organization literature. The concepts of interdependencies and interactions among choices have a long heritage in the organization design literature (e.g. Galbraith 1977; March and Simon 1958; Thompson 1967). However, relatively little analysis has been conducted on the issue of consequences of misperceptions in systems of interconnected choices, and the conditions under which these consequences tend to be large. Some indirect indications of the consequences is provided by research that has explored the concept of "loose coupling" (Glassman 1973; Orton and Weick 1990; Weick 1976; 1982). In loosely coupled systems, the interdependence between activities is low, while in tightly coupled systems, the interdependence is high. For instance, Orton and Weick (1990) note that loose coupling is associated with stability and resistance to change—and presumably to mistakes—since in loosely coupled systems, changes in one part of the system do not propagate to other parts. Conversely, in systems with tight coupling, changes (and errors) tend to spread quickly (Firestone 1985; Wilson and Corbett 1983).

While the previous organization literature has often treated interactions informally, a recent stream of literature has used a formal modeling approach based on the NK-framework, e.g., Levinthal (1997), Levinthal and Warglien (1999), McKelvey (1999), and Rivkin (2000). However, existing work using NKmodels has been silent on what types of relationships arise in their analyses. In a system of $N$ activities, it is assumed that the contribution of any activity to total performance of the system is randomly influenced by $K$ other activities. Thus, no conclusions can be reached with respect to particular types of interaction.

The rest of the paper is organized as follows: In Section II, we study the consequences of a single decision maker misperceiving interaction effects between two activities. Section III covers the case of ignoring interaction effects by a decision maker. Section IV analyzes the case of uncertainty around the 
interaction parameter. In Section V, the model is extended to two managers. Each manager is controlling one activity. We address the question whether it is preferable to have independent or perfectly correlated misperceptions by the managers. We further study the effects of providing one manager with perfect information about the interaction effect. Section VI contains a discussion of the organizational implications of the results and concludes.

\section{Performance declines caused by misperceiving complements and substitutes}

To study formally the effects of misperceiving interactions, a precise definition of interactions and the strength of interactions is needed. We choose to adopt a definition that has been previously used in the literature on teams (Marschak and Radner 1972) and is very consonant with the work on complementarities (Milgrom and Roberts 1990a; Milgrom and Roberts 1992). Let $V$ be a benefit function with arguments $A$ and $B$. Then $A$ and $B$ are said to interact if the level of $A$ affects the marginal benefit of $B$, and vice versa. The interaction between $A$ and $B$ is defined to be stronger than the interaction between $C$ and $D$ if the level of $A$ affects in absolute value the marginal benefit of $B$ more than the level of $C$ affects the marginal benefit of $D$, and vice versa. Thus, if $V$ is a twice-differentiable function, $A$ and $B$ are said to interact if $\frac{\partial^{2} V}{\partial A \partial B} \neq 0 . A$ and $B$ are complements if this cross-partial derivative is positive, i.e., if $V$ is supermodular (Topkis 1978); they are substitutes if the derivative is negative. The interaction between $A$ and $B$ is stronger than the interaction between $C$ and $D$ if $\left|\frac{\partial^{2} V}{\partial A \partial B}\right|>\left|\frac{\partial^{2} V}{\partial C \partial D}\right| . A$ and $B$ are independent if the cross-partial derivative is zero. The basic model contains two activities $A$ and $B$ and one manager M1. The degree of interaction between $A$ and $B$ is measured by the parameter $\alpha$.

Misperceptions of interactions have not been modeled previously, hence the literature does not provide much guidance in this regard. As a natural starting point, we focus on two different misperception structures: additive and multiplicative misperception. In the additive case, M1 misperceives the interaction between $A$ and $B$ to be $(\alpha+\delta)$ rather than $\alpha$. For instance, if the interaction between two 
activities changed, one could think of $(\alpha+\delta)$ as the old interaction, and $-\delta$ as a change that M1 did not recognize. In the multiplicative case, $M 1$ perceives the interaction to be $(1+\delta) \alpha$. Most of the results that are presented below hold for either form of misperception. Consequently, for the sake of exposition, we will focus on the additive case and note in the text when results differ. ${ }^{2}$ (Proofs for both cases can be found in Appendix 2.)

To evaluate the effects of misperception, the case of no misperception, i.e., $\delta=0$, is taken as a benchmark. Let $A^{*}(\alpha, 0)$ and $B^{*}(\alpha, 0)$ be the choices of $A$ and $B$ which maximize the benefit function $V$ and denote with $V(\alpha, 0)$ the ensuing optimal performance. If M1 misperceives the interaction between $A$ and $B, \mathrm{M} 1$ chooses activity levels $A^{*}(\alpha, \delta)$ and $B^{*}(\alpha, \delta)$ that will generally differ from $A^{*}(\alpha, 0)$ and $B^{*}(\alpha, 0)$. Let $V(\alpha, \delta)$ denote the ensuing payoff if the misperception is $\delta$. A measure of the performance decline due to misperception is given by $R(\alpha, \delta)=V(\alpha, \delta)-V(\alpha, 0)$. (All the results are robust to the alternative specification $R(\alpha, \delta)=V(\alpha, \delta) / V(\alpha, 0)$.)

The final part of the model is the specification of the benefit function $V$. Let the benefit of choosing $A$ and $B$ be given by

$V(\alpha, 0)=A+B+\alpha A B-A^{2}-B^{2}$

where the terms $-A^{2}$ and $-B^{2}$ can be thought of as the costs of choosing the levels $A$ and $B .^{3}$ Note, $\frac{\partial^{2} V}{\partial A \partial B}=\alpha$. In words, the larger is $|\alpha|$ the more the level of $A$ affects the marginal benefit of $B$ and vice versa. Thus, $|\alpha|$ measures the strength of the interaction between $A$ and $B$. The sign of $\alpha$ determines the nature of the relationship between $A$ and $B: A$ and $B$ are complements if $\alpha>0$, and substitutes if $\alpha<0$. ${ }^{4}$ Since M1 perceives the interaction to be $(\alpha+\delta)$, M1 chooses $A$ and $B$ to maximize:

$$
V^{l}=A+B+(\alpha+\delta) A B-A^{2}-B^{2}
$$

Straightforward calculation yields that M1's choices are given by

$$
A^{*}(\alpha, \delta)=B^{*}(\alpha, \delta)=\frac{1}{2-\alpha-\delta}
$$


Substituting these choices into the true value function (1) and subtracting $V(\alpha, 0)$ yields

$$
R(\alpha, \delta)=\frac{-\delta^{2}}{(2-\alpha-\delta)^{2}(2-\alpha)}
$$

Figure 1 depicts $\mathrm{R}(\alpha, \delta)$ for two values of $\delta$. This simple figure contains three insights expressed in the following propositions. (For proofs of all Propositions covering both additive and multiplicative misperceptions, see Appendix 2.)

Figure 1: Performance shortfall $\mathrm{R}(\alpha, \delta)$ for the additive case of misperception

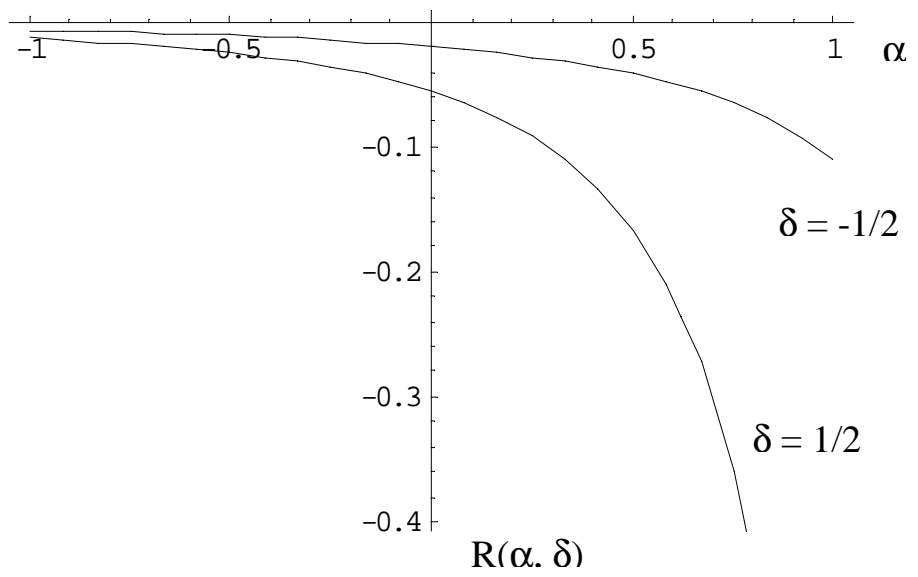

Proposition 1: Given functional assumption (1), for any given misperception modeled additively or multiplicatively, it is less costly to misperceive substitutes than to misperceive (equally strong) complements.

Proposition 1 is illustrated in Figure 1 by picking a pair of symmetric interactions, $\alpha^{\prime}>0$ and $-\alpha^{\prime}<0$ and noting that the performance shortfall for $\alpha^{\prime}>0$ is larger than the performance shortfall for $-\alpha^{\prime}<0$. Given the definition of $R$, Proposition 1 can be interpreted alternatively as, "It is more beneficial to 
eliminate misperceptions for complements than for equally strong substitutes." We can generalize this proposition for any reduction in misperception:

Proposition 1b: Given functional assumption (1), any reduction in misperception leads to a larger benefit for complements than for equally strong substitutes. ${ }^{5}$

The intuition behind this result is that complements tend to amplify the consequences of misperceptions, while substitutes dampen them. For ease of exposition, we will think of $A$ and $B$ as investment levels and start with no misperception $(\delta=0)$. A positive $\delta$ increases M1's assessment of the marginal benefit of $A$, given the current level of $B$, and of the marginal benefit of $B$, given the current level of $A$, leading to (first-order) overinvestments relative to no misperception. Similarly, if $\delta$ is negative, M1 underinvests in $A$ and $B$. Since $A$ and $B$ influence each other's marginal benefit, an additional secondorder effect exists. In the case of complements, a higher level of $A$ further increases the perceived benefit of $B$, leading to a further increase in $B$. In the same way, the higher level of $B$ triggers an optimal upward adjustment of $A$, etc., until the perceived benefits of $A$ and $B$ equal their marginal costs. A similar argument applies for negative misperceptions $(\delta<0)$. In this case, M1's underinvestment in $A$ triggers a further underinvestment in B, etc. Thus, with complements, both first- and second-order effects pull $A$ and $B$ in the same direction away from their optimal values.

In contrast, when $A$ and $B$ are substitutes, the second-order effects have a dampening effect. If $\delta$ is positive, M1 perceives the marginal benefit of $A$ and $B$ to be higher and overinvests. In this case, the higher level of $A$ decreases the marginal benefit of $B$ and leads to a reduction in $B$. Thus, the overinvestment in $B$ is reduced. Similarly, the higher level of $B$ decreases the marginal benefit of $A$ and leads to a reduction in A. (Smaller third-order effects then work in the opposite direction, etc.) Thus, for substitutes, first- and second-order effects work in opposite directions, resulting in a dampening of the consequences of misperceptions. As this argument highlights, second-order effects play a crucial role in the result. Hence, it is important to note that the results derived in this paper may not be directly 
transferable to different set-ups in which second-order effects are less well-behaved due to discontinuities in the underlying functional form.

Proposition 2 establishes that the result of Proposition 1 holds in expectation if $\delta$ is randomly distributed: ${ }^{6}$

Proposition 2: Given functional assumption (1), if misperceptions are randomly distributed, it is less costly, in expectation, to misperceive substitutes than to misperceive (equally) strong complements.

Proposition 2 is illustrated by Figure 1 as follows: pick a value $\alpha^{\prime}>0$. Then, for all values of $\delta$, the corresponding performance decline for $-\alpha^{\prime}$ is lower than that for $\alpha^{\prime}$ (as per Proposition 1). As a result, any weighted sum of all possible performance declines (which is equivalent of assuming some distribution for $\delta$ ) will be smaller for a given $-\alpha^{\prime}$ than the same weighted sum for the corresponding positive $\alpha^{\prime}$.

Many previous discussions of interaction effects have implicitly assumed that stronger interactions always lead to increased fragility. Yet as Figure 1 shows, this need not always be the case.

Proposition 3: For a given misperception, stronger interactions do not always lead to higher performance declines.

Note that while for $\alpha>0$ the performance decline increases as $|\alpha|$ increases, the performance decline actually decreases for $\alpha<0$ as $|\alpha|$ increases. Thus, in this case, for substitutes, the stronger the interaction, the smaller the performance decline given a fixed amount of misperception. In other words, as interactions become stronger, the system becomes more robust if it is composed of substitutes.

It is important to note that the monotonically decreasing relationship between $\alpha$ and the performance decline (as depicted in Figure 1) does not always hold. It hinges on the absence of a "bliss point" with respect to misperceptions. If there exists a value $\alpha^{\prime}$ for which misperceptions do not matter, i.e., for which 
the performance decline is zero, then the performance decline at $\left(\alpha^{\prime}+\varepsilon\right)$ and $\left(\alpha^{\prime}-\varepsilon\right)$, for some positive $\varepsilon$, has to be non-zero. In other words, the relationship between $\alpha$ and the performance decline cannot be monotonic (but has local maximum at $\alpha^{\prime}$ ). For instance, for the case of multiplicative misperceptions, the monotonic relationship is broken. A weaker statement, however, still applies to both the additive and the multiplicative case:

Proposition 3b: Given functional assumption (1), a given misperception causes a larger increase in performance decline when complements become stronger than when substitutes become stronger.

For the additive case, Proposition $3 b$ holds since the performance decline decreases for substitutes and increases for complements as the strength of the interaction increases. For the multiplicative case, the proposition states that the absolute value of the slope of $\mathrm{R}(\alpha, \delta)$ is greater for each $\alpha^{\prime}>0$ than for the corresponding $-\alpha^{\prime}$.

\section{Performance declines caused by ignoring, under- and overestimating interactions}

In this section, we study the effects of ignoring interaction effects, a common type of misperception. In the additive model, ignoring interactions corresponds to a misperception equal to the negative of the true interaction, i.e., $\delta=-\alpha$ (such that $\alpha+\delta=0$ ), while in the multiplicative model it corresponds to $\delta=-1$ (such that $(1+\delta) \alpha=0)$. Since results for both misperception structures are qualitatively similar, we continue to focus on the additive case.

We compare whether ignoring complements is more or less costly than ignoring equally strong substitutes. Formally, $R(\alpha,-\alpha)$, the performance decline due to ignoring complements, is compared to $R(-\alpha, \alpha)$, the performance decline due to ignoring substitutes, for all $\alpha>0$. (Recall, the first argument of $R(\bullet)$ denotes the strength of the interaction, the second argument denotes the misperception.) 
Proposition 4: Given functional assumption (1), ignoring substitutes is less costly than ignoring (equally strong) complements.

Formally, as shown in Appendix 2, $R(\alpha,-\alpha)<R(-\alpha, \alpha)$ for all $\alpha>0$. Ignoring interactions is a specific form of underestimating interactions (interactions are underestimated to the degree that they are ignored). The analysis can be extended to more general underestimations. We will concentrate on "relationship-conserving" underestimations, that is, underestimations for which the manager still perceives substitutes as substitutes and complements as complements. See Figure 2 for an illustration. Points in areas (1) and (3) correspond to relationship-conserving misperceptions, while points on the $45^{\circ}$ line correspond to ignored interactions. For instance, point K corresponds to an ignored substitute, whereas point $\mathrm{K}^{\prime}$ corresponds an ignored complement. As in the case of ignored interactions, we compare underestimations of symmetric interactions. Thus, $R(\alpha,-\delta)$ is compared to $R(-\alpha, \delta)$ with $\alpha>0, \delta>0$, and $\alpha>\delta$. The last inequality states that the misperception is not strong enough to switch the true nature of the relationship.

Figure 2: Ignoring, under-, and overestimating interactions

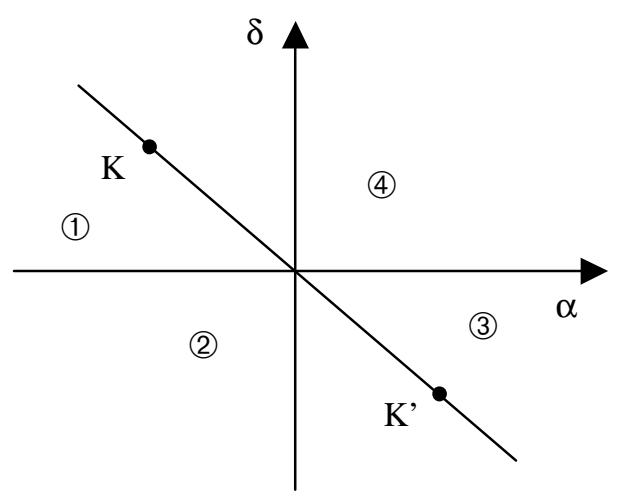

Proposition 5: Given functional assumption (1), underestimating complements (while still perceiving them as complements) is more costly than underestimating (equally strong) substitutes. 
Lastly, the consequences of overestimating complements are compared to the consequences of overestimating substitutes. Again, symmetric pairs of strengths of interaction and misperception (i.e., symmetric points in areas (2) and (4)) are compared to each other.

Proposition 6: Given functional assumption (1), overestimating complements is more costly than (equally) overestimating (equally strong) substitutes.

\section{Performance declines caused by uncertainty around the interaction parameter}

In this section, we analyze misperceptions of the true interaction parameter caused by uncertainty concerning its true value, if the interaction parameter itself is randomly distributed. In its structure, this version of the model comes closest to the models of Athey and Levin (2000) and Persico (2000), who study the value of information given uncertainty around a profit function. However, Athey and Levin's (2000) and Persico's (2000) models contain only one choice variable. Thus, these models do not shed light onto the question whether information with respect to substitute or complementary interactions among choice variables is more valuable.

Consider a manager who receives a noisy signal $z$ of the interaction parameter $\alpha$ both of which she regards as random variables. Denote with $g(\alpha \mid z)$ the conditional distribution of $\alpha$ given signal $z$. The manager thus maximizes over her choices $\boldsymbol{x}$,

$$
V(\alpha \mid z)=\int f(\boldsymbol{x}, \alpha) g(\alpha \mid z) d \alpha
$$

To be more specific, assume the manager's prior belief about $\alpha$ is that $\alpha$ is uniformly distributed over $[-1,1]$. Further assume that the manager receives a signal $z=\alpha+\delta$, with $\delta$ independently and uniformly distributed over $[-1,1]$. The conditional distribution of $\alpha$ given a signal $z$ is then given as follows: if $z \geq 0, \alpha$ is uniformly distributed between $[z-1,1]$, i.e., $g(\alpha \mid z)=1 /(2-z)$. If $z<0, \alpha$ is uniformly 
distributed between $[-1,1+z]$, i.e., $g(\alpha \mid z)=1 /(2+z)$. (Figure 3, displaying all possible values of $(\alpha, z)$, provides an intuition for the conditional probability density functions of $\alpha$ given $z$ and is useful to keep track of the integration boundaries used below.)

Figure 3: All possible values of the signal $z$ and the true interaction parameter $\alpha$

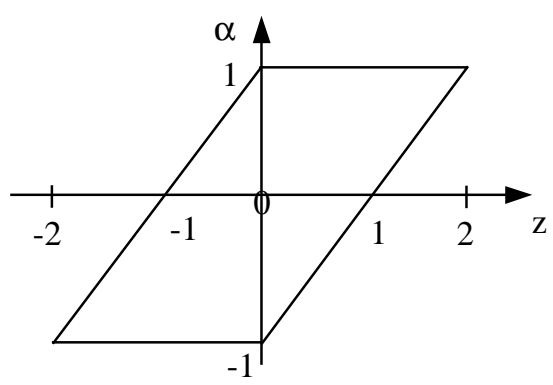

Let $f$ be the quadratic production function used previously. Then, if $z \geq 0$, the manager maximizes over $A$ and $B$ :

$$
V(\alpha \mid z)=\int_{z-1}^{1}\left(A+B+\alpha A B-A^{2}-B^{2}\right) \frac{1}{2-z} d \alpha
$$

If $z<0$, the manager maximizes over $A$ and $B$ :

$$
V(\alpha \mid z)=\int_{-1}^{1+z}\left(A+B+\alpha A B-A^{2}-B^{2}\right) \frac{1}{2+z} d \alpha
$$

Both integrals evaluate to the following expression:

$$
V(\alpha \mid z)=A+B+1 / 2 z A B-A^{2}-B^{2}
$$

(Note, for instance, if $z=2$, the manager knows that $\alpha=1$, since $z$ can take on the value of 2 only if $\alpha=1$ and $\delta=1$. As (5) indicates, if $z=2$, the manager indeed optimizes $V$ using the correct value of $\alpha$, i.e., 1.) Solving (5) for the optimal choices implies that the manager, after receiving signal $z$, will choose

$$
A^{*}=B^{*}=\frac{2}{4-z}
$$


Substituting these choices into $f$ and subtracting the optimal value of $f$ given the true realization of $\alpha$, yields the performance deficit given a signal $z$ and a true realization $\alpha$ :

$$
R(\alpha \mid z)=\frac{(z-2 \alpha)^{2}}{(\alpha-2)(z-4)^{2}}
$$

Similar to the cases studied in previous sections, we want to analyze whether uncertainty about $\alpha$ is more or less detrimental when $\alpha$ is positive or negative, i.e., when $A$ and $B$ are complements or substitutes. In this setting, by focusing on particular values of the signal, we can restrain the underlying interaction parameter. For instance, for $z=1, \alpha$ is uniformly distributed between $[0,1]$, while for $z=-1$, $\alpha$ is uniformly distributed between $[-1,0]$. We can now compute the expected performance loss given $z=1$ (i.e., when $\alpha$ takes on only positive values) and $z=-1$ (i.e., when $\alpha$ takes on only negative values). Substituting $z=1$ into (6) and integrating out $\alpha$ (with integration bounds 0 and 1) yields $2 / 3-\ln (2)=-0.026$. Similarly, substituting $z=-1$ into (6) and integrating out $\alpha$ (with integration bounds -1 and 0 ) yields $2 / 5-\ln (3 / 2)=-0.005$. Thus, similar to previous results, uncertainty around the interaction is more costly when $\alpha$ happens to be negative than when $\alpha$ is positive.

Rather than choosing particular values for $z$, we can also choose ranges of $z$ such that $\alpha$ is restricted to take on only positive or negative values. Note, the probability density function of $z$ is given by: $h(z)=\frac{1}{2}-\frac{1}{4} z$ for $2>z \geq 0$, and $h(z)=\frac{1}{2}+\frac{1}{4} z$ for $-2<z<0$. The most natural cases to compare are $1<z<2$, i.e., values of $z$ that guarantee a positive $\alpha$ (see Figure 3), and $-2<z<-1$, i.e., values of $z$ that guarantee negative values of $\alpha$. (Note the probability of either case is equal to 0.125 .) The expected performance loss when $\alpha$ can only take on positive values is given by:

$$
\int_{1}^{2} \int_{-1+z}^{1} R(\alpha \mid z) \frac{1}{2-z}\left(\frac{1}{2}-\frac{1}{4} z\right) d \alpha d z=\frac{3}{4}+\ln \left(\frac{\sqrt{2}}{3}\right)=-0.0020
$$


The expected performance loss when $\alpha$ can only take on negative values is given by:

$$
\int_{-2}^{0} \int_{-1}^{1+z} R(\alpha \mid z) \frac{1}{2+z}\left(\frac{1}{2}+\frac{1}{4} z\right) d \alpha d z=\ln \left(\frac{108 \sqrt{6}}{125}\right)-\frac{3}{4}=-0.0003
$$

Again we find that uncertainty around the interaction parameter is more detrimental when $\alpha$ happens to be positive than when $\alpha$ is negative.

\section{Misperceptions with two managers}

While the discussion in the previous sections was couched in terms of one manager, an equivalent setup with two managers exists. Manager M1 chooses the level of $A$, and manager M2 chooses the level of $B$. All of the above one-manager models are equivalent to this two-manager set-up if both managers have the same misperception. Thus, both managers believe that the interaction is $(\alpha+\delta)$ rather than $\alpha$. In the following, this assumption is relaxed and the two managers are allowed to have different misperceptions, $\left(\alpha+\delta_{1}\right)$ and $\left(\alpha+\delta_{2}\right)$

With two managers, the true benefit function is still given by (1). M1, who chooses $A$, attempts to maximize, however:

$$
V^{l}=A+B+\left(\alpha+\delta_{1}\right) A B-A^{2}-B^{2}
$$

while M2, who chooses $B$, attempts to maximize:

$$
V^{2}=A+B+\left(\alpha+\delta_{2}\right) A B-A^{2}-B^{2}
$$

By differentiating (7) with respect to $A$, and (8) with respect to $B$, and setting these derivatives equal to zero, we can solve for the Nash-equilibrium choices of M1 and M2: ${ }^{7}$

$$
A^{*}=\frac{2+\alpha+\delta_{1}}{4-\left(\alpha+\delta_{1}\right)\left(\alpha+\delta_{2}\right)} \quad \text { and } \quad B^{*}=\frac{2+\alpha+\delta_{2}}{4-\left(\alpha+\delta_{1}\right)\left(\alpha+\delta_{2}\right)}
$$

Given these choices, the resulting outcome is $V\left(\alpha, \delta_{1}, \delta_{2}\right)$ and the performance decline is defined accordingly as: $R\left(\alpha, \delta_{1}, \delta_{2}\right)=V\left(\alpha, \delta_{1}, \delta_{2}\right)-V(\alpha, 0,0)$. (For more details, see Appendix 2.) 
Stochastic, independent misperceptions

Assume that $\delta_{1}$ and $\delta_{2}$ are independently distributed with probability distribution functions $f\left(\delta_{1}\right)$ and $g\left(\delta_{2}\right)$. The expected performance decline is then given by:

$$
E\left(R\left(\alpha, \delta_{1}, \delta_{2}\right)\right)=\iint R\left(\alpha, \delta_{1}, \delta_{2}\right) f\left(\delta_{1}\right) g\left(\delta_{2}\right) d \delta_{1} d \delta_{2}
$$

As shown in the Appendix, the key results of Section III continue to hold:

Proposition 7: Given functional assumptions (7) and (8), with two managers who have randomly distributed misperceptions:

a) In expectation, it is less costly to misperceive substitutes than to misperceive (equally) strong complements.

b) Stronger interactions do not always lead to higher performance declines.

$\underline{\text { Independent versus perfectly correlated misperceptions }}$

An interesting question to raise is whether independent misperceptions are more costly than perfectly correlated misperceptions. In our set-up, perfectly correlated misperceptions arise when the two managers hold the same belief about the interaction, or when both activities are controlled by the same manager. Two studies have analyzed this question. First, Milgrom and Roberts (1995) show that if the elements of a function $f$ are complements and random errors $\varepsilon_{l}, \ldots, \varepsilon_{n}$ are independently and identically distributed, then $E\left[f\left(x_{1}+\varepsilon_{1}, \ldots, x_{n}+\varepsilon_{n}\right)\right] \leq E\left[f\left(x_{1}+\varepsilon_{1}, \ldots, x_{n}+\varepsilon_{1}\right)\right]$. In words, "when complementarities are present, 'fit' is important, that is, even mistaken variations from a plan are less costly when they are coordinated than when they are made independently" (p. 186). ${ }^{8}$ Note, in Milgrom and Robert's set-up, errors occur directly on the choice variables and not on the perception of the interaction effect.

Second, Crémer $(1990 ; 1993)$ studies the value of independent versus equal misperceptions using a set-up that has a close resemblance to the model analyzed in this paper. Two managers chose independently $A$ and $B$, respectively, resulting in the payoff: 


$$
V=\theta(A+B)+(\alpha-\beta) A B-1 / 2(\alpha+\beta)\left(A^{2}+B^{2}\right)
$$

In contrast to our set-up, in Crémer's model, the interaction terms $\alpha$ and $\beta$ are known. However, uncertainty exists around $\theta$, the parameter on the linear terms, with each manager receiving a signal $\mu=\theta+\varepsilon$, where $\varepsilon$ is randomly distributed. Crémer $(1990 ; 1993)$ finds that it is more beneficial for both managers to receive the same signal $\mu$, if $\alpha>\beta$, i.e., if $A$ and $B$ are complements. If $\alpha<\beta$, i.e., if $A$ and $B$ are substitutes, it is more beneficial for both managers to receive a different signal. Crémer's result is, thus, very similar to the result by Milgrom and Roberts (1995).

As shown in Appendix 2, for uniformly or normally distributed misperceptions of the interaction effect, the reverse result holds in our set-up:

Proposition 8: Given functional assumptions (7) and (8), for complements, independent misperceptions, distributed normally or uniformly, lead to a smaller performance decline than do perfectly correlated misperceptions.

Only for very strong substitutes do independent misperceptions lead to larger performance declines than perfectly correlated misperceptions. This result, thus, offers a cautionary note. In modeling the effects of errors, the assumption of how errors affect choices is not innocuous. It can make a crucial difference whether actions are directly affected or whether interaction effects are misperceived. If managers hold misperceptions with respect to interaction effects, the above result would indicate that if decisions are made on complementary activities, diversity of opinion is more beneficial than "groupthink:" it is preferable to have managers with varied misperceptions than managers who all share the same wrong belief or to have centralized decision making by one mistaken manager. 


\section{One manager with no misperception}

In this section, we analyze the consequences of one manager (M1) having the correct belief about the interaction, while the other manager (M2) still misperceives the interaction to be $\left(\alpha+\delta_{2}\right)$. One can imagine a variety of reasons why M1 might have the correct belief: M1 may simply be smarter than M2, M1 may have received some further training, M1 may have access to superior information with respect to the feedback effects, etc. The main question of this section is whether in the presence of a decision maker who knows the true interaction effects and who, as a consequence, might be able to compensate for errors of the other decision maker, the results with respect to ignoring, over- and underestimating interaction effects (Propositions 4-6) continue to hold. Proposition 9 establishes that this is indeed the case:

Proposition 9: Given functional assumptions (7) and (8) and given that M1 acts according to the true interaction, it is

a) more costly for M2 to ignore complements than substitutes

b) more costly to underestimate complements than substitutes

c) more costly to overestimate complements than substitutes

\section{Discussion and conclusion}

This paper set out to make a first attempt at formally studying the implications of misperceiving different types of interactions between activity choices. The overall flavor of the results we derived is that misperceptions with respect to complements are more costly than misperceptions with respect to substitutes. While the specific results were derived using a particular functional form, and thus await a more general analysis, the results show the importance of distinguishing between different types of interactions. Previous work frequently distinguished only between "interaction" and "independence." As the results in this paper show, complementary interactions behave, however, quite differently from substitute interactions. Thus, an explicit treatment of these two types of interactions can yield new insights. 
With the caveat in mind that the results concerning complementary and substitute interactions have only been shown in the context of a functional form that has well-behaved continuous second-order effects, we want to outline, nevertheless, a few implications of the central results. Since many organizational choices can affect the degree of misperception, or conversely the degree of information about interaction effects, the result that misperceptions of complementary interactions tend to be more detrimental than misperceptions of substitute interactions is of particular interest. For instance, while the simplification strategies outlined in the first section of this paper can lead to misperceptions of interaction effects, they also tend to have benefits associated with them. Thus, firms need to assess the trade-off between the benefits of these strategies and the costs due to misperceptions. Our analysis casts some light onto the cost side of this trade-off. Consider the effect of profit-center incentives. On one hand, profitcenter incentives allow high-powered incentives for division managers. At the same time, the benefits due to tighter accountability have to be traded off against the potential performance losses due to ignored interactions that are also caused by profit-center incentives. Our results suggest that if the investments undertaken in a division decrease the marginal benefit of investments in other divisions, the performance loss due to ignored interactions tend to be small. If the investments increase the marginal benefit of investments of other divisions, however, the cost of having strong profit-center incentives might be very large.

A further intra-organizational implication of our results is that "better" managers (in this case, managers who have smaller misperceptions) should be assigned to decisions that have strong complementary interactions. Similarly, the payoff from information-gathering for problems that are strongly complementary, for instance, through the use of cross-functional teams, and the benefits from training managers who have to make these decisions, are likely to be high.

In a similar vein, decisions that change the strength of interactions between activities need to be clearly communicated, in particular if it is a strengthening (or a creation) of a complementary relationship. Consider, for example, a firm for which speed-to-market is very important (payoffs to reducing time-to-market are convex). Further consider an investment in information technology that 
allows the design department of this firm to communicate ideas much faster to the production department than previously possible. This investment strengthens the positive interdependence between investments in design speed and investments in flexible production technology. (The marginal benefit of increasing design speed is higher when the production technology is flexible.) Moreover, this complementarity has been strengthened by the investment in information technology. If the managers overseeing investments in design and production are not fully aware of the increase in positive interdependence, they will systematically underinvest in their respective departments, incurring potentially large performance deficits.

In contrast, misperceptions with respect to the interaction of substitute investments tend to have smaller performance implications. For instance, consider a manager making investment decisions in the context of moving a new product from design to manufacturing. Investment choices include upgrading the $\mathrm{CAD}$ equipment and the amount of training provided to technicians who use the preliminary engineering drawings to build prototypes, in the process of which changes are made to the drawings and the new product itself as the technicians discover ways to make the product easier to manufacture (Bechky 2000). Investments in upgraded CAD equipment that is able to test for manufacturability itself would reduce the marginal benefit of investing in highly skilled technicians, and vice versa. Thus, the investments interact as substitutes. As our results suggest, misperceiving the precise strength of this interaction when making investment decisions may not be very costly.

Since organizational design has an impact on information flow within firms (Henderson and Clark 1990), the results point to potentially useful—if rough—suggestions for designs that can help alleviate problems caused by misperceptions. As von Hippel (1990) reports, companies frequently do not take interdependencies among activities into account when tasks are partitioned. Our model suggests that if grouping together choices reduces the degree of misperception, strong complements should be grouped together since the benefit of reducing the misperception tends to be high.

The asymmetry result with respect to costs of misperceiving complements and substitutes has also implications beyond the intra-organizational sphere. If information about the interaction between 
decisions (e.g., between design parameter choices of a particular product) is better gained within a firm than across firms (Afuah 1998; Kogut and Zander 1992; Sridhar and Balachandran 1997), and decisions affect each other in a complementary manner, the entire set of decisions should better be kept within one firm. However, choices that interact as substitutes may be kept in separate organizations, because potential problems caused by misperceptions of interactions tend to be small.

This paper started out by expressing a concern with competitive advantage that is based on systems of tightly interconnected choices: if these systems are very fragile, then the competitive advantage would be very fragile, too. As the analysis has shown, there is some justification for the concern. Systems composed of complements tend to be fragile. However, substitutes play an important stabilizing role that has not received much attention in the recent literature. Thus, as managers are constructing and managing systems of interconnected choices, they should become aware of both the potential problems arising from systems of complements (so that they can take counter-measures) and of the potential benefits of substitutes.

This paper clearly provided only a first step in the analysis of misperceptions of different types of interaction effects. Possible useful extensions include a more explicit model of how managers form their beliefs/perceptions of interaction effects and how they update their beliefs as they receive feedback from the environment. Imposing more structure on the interaction effects in simulation models may provide another fruitful path to analyze the organizational design implications of misperceptions. 


\section{Appendix 1: Definitions}

To state a more general case of the monotone comparative statics result, we need to introduce some notation. A lattice $(X, \geq)$ is a set $X$ with a partial order $\geq$ with the property that for any $x$ and $y$ in $X, X$ also contains a smallest element under the order that is larger than both $x$ and $y$ and a largest element that is smaller than both. Let $x \vee y$ denote the smallest element larger than $x$ and $y$, and $x \wedge y$ denote the largest element smaller than $x$ and $y$. A sublattice of a lattice $X$ is a subset $S$ of $X$, if for any $x, y \in S,(x \wedge y) \in S$, and $(x \vee y) \in S$. Given a real-valued function $f$ on a lattice $X, f$ is called supermodular if and only if for any $x$ and $y$ in $X: f(x)-f(x \wedge y) \leq f(x \vee y)-f(y)$.

Furthermore, a function $f$ has increasing differences in $(x ; \theta)$ if for $x^{\prime} \geq x, f\left(x^{\prime}, \theta\right)-f(x, \theta)$ is monotone nondecreasing in $\theta$. With these definitions, Topkis' (1978) main comparative static result can be stated as follows: Let $X$ be a lattice, $\Theta$ a partially ordered set, and $f: X \times \Theta \rightarrow \Re$. Let $x^{*}(\theta)$ be the set of maximizers of $f(x, \theta)$ subject to $x \in S$, with $S$ a sublattice of $X$. If $f(x, \theta)$ is supermodular in $x$ and has increasing differences in $(x ; \theta)$, then $x^{*}(\theta)$ is monotone nondecreasing in $\theta$.

\section{Further definitions:}

- A function $f$ is quasi-supermodular if $f(x) \geq f(x \wedge y)$ implies $f(x \vee y) \geq f(y)$ and $f(x)>f(x \wedge y)$ implies $f(x \vee y)>f(y)$.

- A positive function is log-supermodular if the logarithm of that function is supermodular.

- A function $f$ satisfies the single-crossing property in $(x, \theta)$ if for $x^{\prime}>x, \theta^{\prime}>\theta, f\left(x^{\prime}, \theta\right)>f(x, \theta)$ implies $f\left(x^{\prime}, \theta^{\prime}\right)>f(x, \theta)$, and $f\left(x^{\prime}, \theta\right) \geq f(x, \theta)$ implies $f\left(x^{\prime}, \theta^{\prime}\right) \geq f(x, \theta)$.

- Assume there is a finite set of random processes $s_{i}^{t} \in S_{i}$, where $S_{i} \subseteq \mathfrak{R}$ is locally compact and letting $S=$ $S_{1} \times \ldots \times S_{m}$ be the ordered set defined by the vector ordering. A random process is Markovian if $\left(s_{i}^{t} \mid s^{t-1}, s^{t-2}, \ldots\right)=\left(s_{i}^{t} \mid s^{t-1}\right)$. A random process has positive persistence of uncertainty if $s^{t-1}>v^{t-1}$ for all $i$ implies that $\left(s_{i}^{t} \mid s^{t-1}\right)$ is equal are larger in the sense of first order stochastic dominance than $\left(s_{i}^{t} \mid v^{t-1}\right)$. 


\section{Appendix 2: Proofs of propositions}

In the additive case (AC), a manager perceives the interaction to be $(\alpha+\delta)$ rather than $\alpha$. In the multiplicative case $(\mathrm{MC})$, the manager perceives $(1+\delta) \alpha$. For second-order conditions to be fulfilled, it is assumed that $|\alpha|<2,|\alpha+\delta|<2$ (for AC), and $|(1+\delta) \alpha|<2$ (for MC). We also focus on relationshipconserving misperceptions, i.e., $\alpha+\delta>0$ if $\alpha>0$, and $\alpha+\delta<0$ if $\alpha<0$ (for AC) and $\delta \geq-1$ (for MC). For compactness, it is useful to compute the expressions for the performance declines for the general twomanager case and treat the one-manager case as a special case with $\delta=\delta_{1}=\delta_{2}$.

For $\mathrm{AC}: \quad \mathrm{R}\left(\alpha, \delta_{1}, \delta_{2}\right)=-\frac{(2+\alpha) \delta_{2}^{2}+(2+\alpha) \delta_{1} \delta_{2}\left(\alpha+\delta_{2}\right)+\delta_{1}^{2}\left(2+\alpha+2 \delta_{2}+\alpha \delta_{2}+\delta_{2}^{2}\right)}{(2-\alpha)\left(4-\left(\alpha+\delta_{1}\right)\left(\alpha+\delta_{2}\right)\right)^{2}}$

For MC: $\mathrm{R}\left(\alpha, \delta_{1}, \delta_{2}\right)=-\frac{\alpha^{2}\left((2+\alpha) \delta_{2}^{2}+\alpha(2+\alpha) \delta_{1} \delta_{2}\left(1+\delta_{2}\right)+\delta_{1}^{2}\left(2+\alpha\left(1+\delta_{2}\left(2+\alpha+\alpha \delta_{2}\right)\right)\right)\right)}{(2-\alpha)\left(4-\alpha^{2}\left(1+\delta_{1}\right)\left(1+\delta_{2}\right)\right)^{2}}$

Proposition 1: For any given misperception, it is less costly to misperceive substitutes than to misperceive (equally strong) complements: $\quad \mathrm{R}(-\alpha, \delta)-\mathrm{R}(\alpha, \delta)>0$, for all $\alpha>0$ and all $\delta$

AC: using (A1) with $\delta=\delta_{1}=\delta_{2}$ yields: $\frac{\delta^{2}}{(2-\alpha)(2-\alpha-\delta)^{2}}-\frac{\delta^{2}}{(2+\alpha)(2+\alpha-\delta)^{2}}>0$

MC: using (A2) with $\delta=\delta_{1}=\delta_{2}$ yields: $\frac{\alpha^{2} \delta^{2}}{(2-\alpha)(2-\alpha-\alpha \delta)^{2}}-\frac{\alpha^{2} \delta^{2}}{(2+\alpha)(2+\alpha+\alpha \delta)^{2}}>0$

In both cases, the denominator of the second term is larger than that of the first term.

Proposition 1b: Claim 1: Reducing $|\delta|$ is always beneficial: $\frac{\partial \mathrm{V}}{\partial \delta}>0$ for $\delta<0 ; \frac{\partial \mathrm{V}}{\partial \delta}<0$ for $\delta>0$

Claim 2: Marginal changes of $\delta$ lead to larger performance changes for complements than for equally

strong substitutes: $\left|\frac{\partial \mathrm{V}(\alpha, \delta)}{\partial \delta}\right|>\left|\frac{\partial \mathrm{V}(-\alpha, \delta)}{\partial \delta}\right|$ for $\alpha>0$

$\mathrm{AC}: \frac{\partial \mathrm{V}(\alpha, \delta)}{\partial \delta}=\frac{-2 \delta}{(2-\alpha-\delta)^{3}} \quad$ Claims 1 and 2 follow by inspection 
MC: $\frac{\partial \mathrm{V}}{\partial \delta}(\alpha, \delta)=\frac{-2 \alpha^{2} \delta}{(2-\alpha-\alpha \delta)^{3}}$ Claim 1 follows by inspection. For Claim 2 recall that $\delta \geq-1$. Claim 2 holds with equality for $\delta=-1$ and strict inequality for $\delta>-1$.

Proposition 2: If misperceptions are randomly distributed, it is less costly to misperceive substitutes than to misperceive (equally) strong complements: Let $\delta$ be a continuous random variable with probability density function $f(\delta)$ with a support that is appropriately restricted so that second-order conditions for the maximization problem are satisfied. Then,

$$
\int(R(-\alpha, \delta)-R(\alpha, \delta)) f(\delta) d \delta>0 \quad \text { for all } \alpha>0
$$

Proposition 2 follows directly from Proposition 1 since $\mathrm{R}(-\alpha, \delta)-\mathrm{R}(\alpha, \delta)$ is positive for all $\delta$. C

Proposition 3: Stronger interactions do not always lead to higher performance declines:

Substituting $\delta=\delta_{1}=\delta_{2}$ into (A1) and differentiating with respect to $\alpha$ yields

$\frac{\partial R(\alpha, \delta)}{\partial \alpha}=\frac{-\delta^{2}(6-3 \alpha-\delta)}{(2-\alpha)^{2}(2-\alpha-\delta)^{3}}<0 \quad$ for all $\alpha$ and all $\delta$

Thus, for the additive case, if $\alpha<0$, the performance decline decreases as $|\alpha|$ increases.

Proposition 3b: For complements, stronger interactions lead to larger performance declines than for

substitutes: $\quad \frac{\partial \mathrm{R}(\alpha, \delta)}{\partial \alpha}+\frac{\partial \mathrm{R}(-\alpha, \delta)}{\partial \alpha}<0$ for all $\alpha>0$

For AC, Proposition 3b follows directly from (A3).

For MC, the LHS of (A4) evaluates to: $\frac{8+2 \alpha-\alpha^{2}(1+\delta)}{(2+\alpha)^{2}(2+\alpha+\alpha \delta)^{3}}-\frac{8-2 \alpha-\alpha^{2}(1+\delta)}{(2-\alpha)^{2}(2-\alpha-\alpha \delta)^{3}}$

(A5) is decreasing in $\delta$ and achieves its highest value in the admissible range of $\delta$ at $\delta=-1$. After substituting $\delta=-1$ into (A5), straightforward calculation shows that (A5) is negative for all $\alpha>0$. 
Propositions 4-6: Ignoring (under- and overestimating) substitutes is less costly than ignoring (underand overestimating) equally strong complements:

for $\mathrm{AC}: \mathrm{R}(-\alpha, \delta)-\mathrm{R}(\alpha,-\delta)>0$ for $\alpha>0$

for $\mathrm{MC}: \mathrm{R}(-\alpha, \delta)-\mathrm{R}(\alpha, \delta)>0$ for $\alpha>0$

Proposition 4 (ignoring interactions) covers the case $\delta=\alpha(\mathrm{AC})$ or $\delta=-1$ (MC)

Proposition 5 (relationship-conserving underestimation): $0<\delta<\alpha$ (AC) or $-1<\delta<0$ (MC)

Proposition 6 (overestimation): $\delta<0(\mathrm{AC})$ or $\delta>0(\mathrm{MC})$

Evaluating (A6) yields: $\frac{\delta^{2}}{(2-\alpha)(2-\alpha+\delta)^{2}}-\frac{\delta^{2}}{(2+\alpha)(2+\alpha-\delta)^{2}}$

(A8) is decreasing in $\delta$ and achieves its lowest value in the admissible range of $\delta$ at $\delta=\alpha$. Straightforward calculation shows that (A8) is positive for all $\alpha>0$ if $\delta=\alpha$. Hence, (A8) is positive for all $\delta \leq \alpha$, covering Propositions 4-6 for AC. (C)

Evaluating (A7) yields: $\frac{\alpha^{2} \delta^{2}}{(2-\alpha)(2-\alpha-\alpha \delta)^{2}}-\frac{\alpha^{2} \delta^{2}}{(2+\alpha)(2+\alpha+\alpha \delta)^{2}}$

(A9) is increasing in $\delta$ and achieves its lowest value in the admissible range of $\delta$ at $\delta=-1$.

Straightforward calculation shows that (A9) is positive for all $\alpha>0$ if $\delta=-1$. Hence, (A9) is positive for all $\delta \geq-1$, covering Propositions 4-6 for MC. 두

Proposition 7: Propositions 2 and 3 hold for the two-manager case:

Proposition 7a: First, it can be shown (for both AC and MC), analogously to Proposition 1, that $\mathrm{R}\left(-\alpha, \delta_{1}, \delta_{2}\right)-\mathrm{R}\left(\alpha, \delta_{1}, \delta_{2}\right) \geq 0$ for all $\alpha \geq 0$, and all $\delta_{1}, \delta_{2}$.

(To ensure that second-order conditions are met, we further restrict $0 \leq \alpha \leq 1,-1<\delta_{1}<1,-1<\delta_{2}<1$ ).

(A10) holds since it can be shown that (A10) has a local minimum at $\delta_{1}=\delta_{2}=0$ taking a value of 0 . Since (A10) holds for any $\delta_{1}, \delta_{2}$, integrating (A10) over $\delta_{1}$ and $\delta_{2}$ given any probability density function of $\delta_{1}$ and $\delta_{2}$ (with appropriate support) will yield a positive number (analogously to Proposition 2). 
Proposition 7b: An example will suffice to show that stronger interactions do not always lead to larger performance declines. Let $\delta_{1}, \delta_{2}$, be identically and independently distributed with $\delta_{1} \sim \mathrm{u}[-1,1]$ and $\delta_{2} \sim \mathrm{u}[-1,1]$. Then,

$$
\mathrm{E}\left(\mathrm{R}\left(\alpha, \delta_{1}, \delta_{2}\right)\right)=\int_{-1}^{1} \int_{-1}^{1} R\left(\alpha, \delta_{1}, \delta_{2}\right) \frac{1}{2} \frac{1}{2} d \delta_{1} d \delta_{2}
$$

In Figure A-1, (A11) is plotted as a function of $\alpha$ for $\mathrm{AC}$ (To ensure that second-order conditions are fulfilled, $\alpha$ is confined to the interval $[-1,1]$.) As Figure A-1 indicates, for substitutes an increase in the strength of interactions leads to a reduction in the performance decline.

Proposition 8: For uniformly or normally distributed misperceptions, independent misperceptions lead to a smaller performance decline than do perfectly correlated misperceptions in the case of complements.

Figure A-1 includes a graph of $\mathrm{E}(\mathrm{R}(\alpha, \delta, \delta))$, i.e., the expected performance decline given stochastic but perfectly correlated misperceptions and a graph of (A11), the expected performance decline given independently distributed misperceptions.

Figure A-1

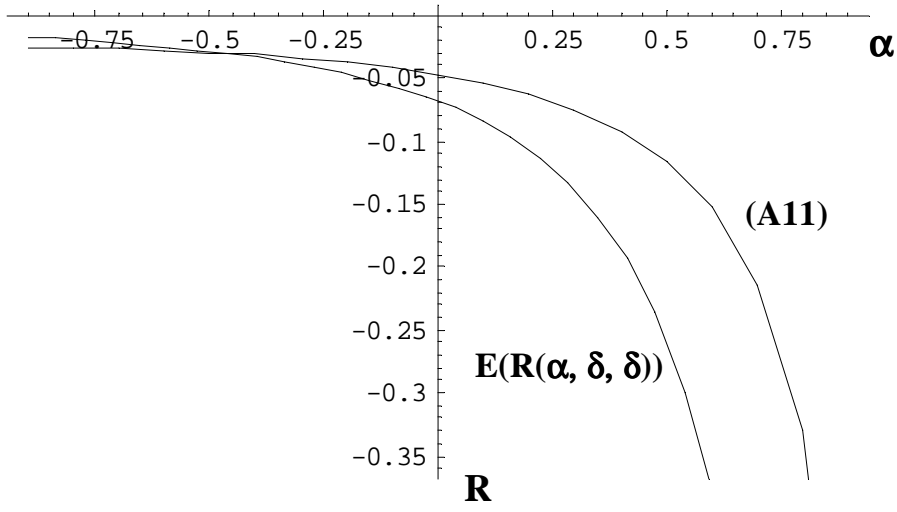

As the Figure indicates, for $\alpha>0$, independent misperceptions lead to a smaller performance decline, while for strong substitutes, perfectly correlated errors lead to a smaller performance decline. For MC, (A11) has a maximum at $\alpha=0$ and crosses $\mathrm{E}(\mathrm{R}(\alpha, \delta, \delta))$ at $\alpha=0$. Thus independent misperceptions lead to a smaller performance decline than perfectly correlated misperceptions for $\alpha>0$ and to a larger 
performance decline for $\alpha<0$. If the misperceptions are assumed to follow a standard normal distribution truncated at -1 and 1, i.e., $\delta \sim \phi(\delta) /(1-2 \Phi(-1)),-1<\delta<1$, with $\phi(\cdot)$ the standard normal probability density function and $\Phi(\cdot)$ the standard normal cumulative density function, similar results are obtained (more details available from the author).

Proposition 9: Propositions 4-6 hold with M1 having no misperception.

A similar approach as in proving Propositions 4-6 is followed. We need to show:

for AC: $\mathrm{R}\left(-\alpha, 0, \delta_{2}\right)-\mathrm{R}\left(\alpha, 0,-\delta_{2}\right)>0$ for $\alpha>0$

for MC: $\mathrm{R}\left(-\alpha, 0, \delta_{2}\right)-\mathrm{R}\left(\alpha, 0, \delta_{2}\right)>0$ for $\alpha>0$

Evaluating (A12) yields: $\frac{(2+\alpha) \delta_{2}^{2}}{(2-\alpha)\left(4-\alpha^{2}+\alpha \delta_{2}\right)^{2}}-\frac{(2-\alpha) \delta_{2}^{2}}{(2+\alpha)\left(4-\alpha^{2}+\alpha \delta_{2}\right)^{2}}$

Evaluating (A13) yields: $\frac{\alpha^{2}(2+\alpha) \delta_{2}^{2}}{(2-\alpha)\left(4-\alpha^{2}\left(1+\delta_{2}\right)\right)^{2}}-\frac{\alpha^{2}(2-\alpha) \delta_{2}^{2}}{(2+\alpha)\left(4-\alpha^{2}\left(1+\delta_{2}\right)\right)^{2}}$

In both (A14) and (A15) the first term has a larger positive numerator and a smaller positive denominator for all $\delta$. Hence, both (A14) and (A15) are positive for all $\delta$. 


\section{$\underline{\text { References }}$}

Afuah, A., "Dynamic Boundaries of the Firm: Are Firms Better Off Being Vertically Integrated in the Face of a Technological Change?," University of Michigan Business School, Ann Arbor, 1998.

Athey, S., "Monotone Comparative Statics under Uncertainty," Quarterly Journal of Economics, (forthcoming-a).

Athey, S., "Single Crossing Properties and the Existence of Pure Strategy Equilibria in Games of Incomplete Information," Econometrica, (forthcoming-b).

Athey, S. and J. Levin, "The Value of Information in Monotone Decision Problems," working paper, MIT, Cambridge, 2000.

Baligh, H. H., R. M. Burton, and B. Obel, "Organizational Consultant: Creating a Useable Theory for Organizational Design,” Management Science, 42, (1996), 1648-1662.

Banker, R. D. and A. A. Atkinson, Management Accounting, Prentice Hall, Englewood Cliffs, NJ, 1996.

Bechky, B., "Sharing Meaning Across Occupational Communities: The Transformation of Knowledge on a Production Floor," working paper, University of Pennsylvania, Wharton School, Philadelphia, 2000.

Blackwell, D., "Comparisons of Experiments," Proceedings of the Second Berkeley Symposium on Mathematical Statistics, (1951), 93-102.

Blackwell, D., "Equivalent Comparisons of Experiments,” Annals of Mathematical Statistics, 24, (1953), $265-272$.

Bowman, E. H., "Consistency and Optimality in Managerial Decision Making," Management Science, 9, (1963), 310-321.

Burnett, W. M., B. G. Silverman, and D. J. Monetta, "R\&D Project Appraisal at the Gas Research Institute," Operations Research, 41, (1993), 1020-1032.

Burton, R. M. and B. Obel, Designing Efficient Organizations: Modelling and Experimentation, NorthHolland, Amsterdam, 1984.

Carley, K. M., “Organizational Learning and Personnel Turnover," Organization Science, 3, (1992), 20 46.

Carley, K. M. and Z. Lin, “A Theoretical Study of Organizational Performance Under Information Distortion," Management Science, 43, (1997), .

Clark, K. B. and T. Fujimoto, Product Development Performance: Strategy, Organization, and Management in the World Auto Industry, Harvard Business School Press, Boston, 1991.

Cooper, R. and R. S. Kaplan, "Measure Cost Right: Make the Right Decisions," Harvard Business Review, 66, September-October, (1988), 96-103.

Crémer, J., "Common Knowledge and the Co-ordination of Economic Activities," in M. Aoki, B. Gustafsson, and O. Williamson (Eds.), The Firm as a Nexus of Treaties, Sage, London, 1990. 
Crémer, J., "Corporate Culture and Shared Knowledge," Industrial and Corporate Change, 2, (1993), $351-386$.

Dearden, J., "Measuring Profit Center Managers," Harvard Business Review, 65, September-October, (1987), 84-88.

Diehl, E. and J. D. Sterman, "Effects of Feedback Complexity on Dynamic Decision Making," Organizational Behavior and Human Decision Processes, 62, (1995), 198-215.

Drazin, R. and A. H. Van de Ven, "Alternative Forms of Fit in Contingency Theory," Administrative Science Quarterly, 30, (1985), 514-539.

Edlin, A. S. and C. Shannon, "Strict Monotonicity in Comparative Statics," Journal of Economic Theory, 81, (1998), 201-219.

Eppinger, S. D., D. E. Whitney, R. P. Smith, and D. A. Gebala, "A Model-Based Method for Organizing Tasks in Product Development," Research in Engineering Design, 6, (1994), 1-13.

Firestone, W. A., "The Study of Loose Coupling: Problems, Progress, and Prospects," Research in Sociology of Education and Socialization, 5, (1985), 3-30.

Friedman, E. J. and S. Johnson, "Dynamic Monotonicity and Comparative Statics for Real Options," Journal of Economic Theory, 75, (1997), 104-121.

Galbraith, J. R., Organization Design, Addison-Wesley, Reading, MA, 1977.

Garud, R. and A. Kumaraswamy, "Technological and Organizational Designs for Realizing Economies of Substitution,” Strategic Management Journal, 16, (1995), 93-109.

Geanakoplos, J. and P. R. Milgrom, "A Theory of Hierarchies Based on Limited Managerial Attention," Journal of the Japanese and International Economies, 5, (1991), 205-225.

Glassman, R. B., "Persistence and Loose Coupling in Living Systems," Behavioral Science, 18, (1973), 83-98.

Groves, T. and R. Radner, “Allocation of Resources in a Team,” Journal of Economic Theory, 4, (1972), 415-441.

Gulati, R. and H. Singh, "The Architecture of Cooperation: Managing Coordination Costs and Appropriation Concerns in Strategic Alliances," Administrative Science Quarterly, 43, (1998), 781814.

Hall, D. L. and A. Nauda, "An Interactive Approach for Selecting R\&D Projects," IEEE Transactions Engineering Management, 37, (1990), 126-133.

Hambrick, D. C. and P. A. Mason, "Upper Echelons: The Organization as a Reflection of Its Top Managers," Academy of Management Review, 9, (1984), 193-206.

Hayes, R. H. and R. Jaikumar, "Manufacturing's Crisis: New Technologies, Obsolete Organizations," Harvard Business Review, 66, September-October, (1988), 77-85. 
Henderson, R. M., "Underinvestment and Incompetence as Responses to Radical Innovation: Evidence from the Photolithographic Alignment Equipment Industry," RAND Journal of Economics, 24, (1993), 248-270.

Henderson, R. M. and K. B. Clark, “Architectural Innovation: The Reconfiguration of Existing Product Technologies and the Failure of Established Firms," Administrative Science Quarterly, 35, (1990), 9-30.

Jaikumar, R., "Postindustrial Manufacturing," Harvard Business Review, 64, 6, (1986), 69-76.

Jewitt, I., "Choosing Between Risky Prospects: The Characterization of Comparative Statics Results, and Location Independent Risk," Management Science, 35, (1989), 60-70.

Kaplan, R. S. and D. P. Norton, "The Balanced Scorecard - Measures that Drive Performance," Harvard Business Review, 70, January-February, (1992), 71-79.

Kiesler, S. B. and L. Sproull, "Managerial Response to Changing Environments: Perspectives on Problem Sensing from Social Cognition," Administrative Science Quarterly, 27, (1982), 548-570.

Kogut, B. and U. Zander, "Knowledge of the Firm, Combinative Capabilities, and the Replication of Technology," Organization Science, 3, (1992), 383-397.

Kremer, M., "The O-ring Theory of Economic Development," Quarterly Journal of Economics, 108, (1993), 551-575.

Kremer, M. and E. Maskin, Working paper No. 5718, National Bureau of Economic Research, Cambridge, MA, 1996.

Lehman, E. L., “Comparing Location Experiments,” Annals of Statistics, 16, (1988), 521-533.

Levinthal, D. A., “Adaptation on Rugged Landscapes,” Management Science, 43, (1997), 934-950.

Levinthal, D. A. and M. Warglien, "Landscape Design: Designing for Local Action in Complex Worlds," Organization Science, 10, (1999), 342-357.

MacDuffie, J. P., "Human Resource Bundles and Manufacturing Performance: Organizational Logic and Flexible Production Systems in the World Automobile Industry," Industrial and Labor Relations Review, 48, (1995), 197-221.

Malone, T. W., "Modeling Coordination in Organizations and Markets," Management Science, 33, (1987), 1317-1332.

March, J. G. and H. A. Simon, Organizations, John Wiley \& Sons, New York, 1958.

Marschak, J. and R. Radner, Economic Theory of Teams, Yale University Press, New Haven, 1972.

McKelvey, B., “Avoiding Complexity Catastrophe in Coevolutionary Pockets: Strategies for Rugged Landscapes," Organization Science, 10, (1999), 294-321.

Milgrom, P. R. and J. Roberts, “The Economics of Modern Manufacturing: Technology, Strategy, and Organization," American Economic Review, 80, (1990a), 511-528. 
Milgrom, P. R. and J. Roberts, "Rationalizability, Learning, and Equilibrium in Games with Strategic Complementarities," Econometrica, 58, (1990b), 1255-1277.

Milgrom, P. R. and J. Roberts, Economics, Organization, and Management, Prentice Hall, Englewood Cliffs, NJ, 1992.

Milgrom, P. R. and J. Roberts, "Complementarities and Fit: Strategy, Structure, and Organizational Change in Manufacturing," Journal of Accounting and Economics, 19, (1995), 179-208.

Milgrom, P. R. and C. Shannon, "Monotone Comparative Statics," Econometrica, 62, (1994), 157-180.

Miller, D. and P. H. Friesen, Organizations: A Quantum View, Prentice Hall, Englewood Cliffs, NJ, 1984.

Murmann, J. P. and M. L. Tushman, "Organizational Responsiveness to Environmental Shock as an Indicator of Organizational Foresight and Oversight: The Role of Executive Team Characteristics and Organizational Context," in R. Garud, P. R. Nayyar, and Z. B. Shapira (Eds.), Technological Innovation, Cambridge University Press, New York, 1997.

Nelson, R. R. and S. G. Winter, Jr., An Evolutionary Theory of Economic Change, Harvard University Press, Cambridge, MA, 1982.

Nemetz, P. and L. W. Fry, "Flexible Manufacturing Organizations: Implications for Strategy Formulation and Organizations Design," Academy of Management Review, 13, (1988), 626-638.

Orton, J. D. and K. E. Weick, "Loosely Coupled Systems: A Reconceptualization," Academy of Management Review, 15, (1990), 203-223.

Persico, N., "Information Acquisition in Auctions," Econometrica, 68, (2000), 135-148.

Porter, M. E., "What is Strategy?," Harvard Business Review, 74, 6, (1996), 61-78.

Porter, M. E. and N. Siggelkow, "Contextuality Within Activity Systems," working paper, Reginald H. Jones Center for Management Strategy, Policy and Organization, University of Pennsylvania, Philadelphia, 2001.

Radner, R., "Teams," in C. B. McGuire, and R. Radner (Eds.), Decision and Organization, NorthHolland, Amsterdam, 1972.

Reece, J. S. and W. R. Cool, "Measuring Investment Center Performance," Harvard Business Review, 56, May-June, (1978), 1-11.

Rivkin, J. W., "Imitation of Complex Strategies,” Management Science, 46, (2000), 824-844.

Schaefer, S., "Product Design Partitions with Complementary Components," Journal of Economic Behavior and Organization, 38, (1999), 311-330.

Shannon, C., "Weak and Strong Monotone Comparative Statics," Economic Theory, 5, (1995), 209-227.

Siggelkow, N., "Change in the Presence of Fit: The Rise, the Fall, and the Renascence of Liz Claiborne," Academy of Management Journal, (forthcoming).

Simon, H. A., Models of Man: Social and Rational; Mathematical Essays on Rational Human Behavior in a Social Setting, John Wiley, New York, 1957. 
Sridhar, S. S. and B. V. Balachandran, "Incomplete Information, Task Assignment, and Managerial Control Systems," Management Science, 43, (1997), 764-778.

Steele, L. W., "Selecting R\&D Programs and Objectives," Research Technology Management, 31, March-April, (1988), 17-36.

Sterman, J. D., "Misperceptions of Feedback in Dynamic Decision Making," Organizational Behavior and Human Decision Processes, 43, (1989a), 301-335.

Sterman, J. D., "Modeling Managerial Behavior: Misperceptions of Feedback in a Dynamic Decision Making Experiment," Management Science, 35, (1989b), 322-339.

Sterman, J. D., "Learning In and About Complex Systems," System Dynamics Review, 10, (1994), 291330.

Thompson, J. D., Organizations in Action, McGraw-Hill, New York, 1967.

Topkis, D. M., "Minimizing a Submodular Function on a Lattice," Operations Research, 26, (1978), 305321.

Ulrich, K. and V. Krishnan, "Product Development Decisions: A Review of the Literature," Management Science, 47, (2001), 1-21.

Vives, X., "Nash Equilibrium with Strategic Complementarities," Journal of Mathematical Economics, 19, (1990), 305-321.

von Hippel, E., "Task Partitioning: An Innovation Process Variable," Research Policy, 19, (1990), 407418.

Weick, K. E., "Educational Organizations as Loosely Coupled Systems," Administrative Science Quarterly, 21, (1976), 1-19.

Weick, K. E., "Management of Organizational Change Among Loosely Coupled Elements," in P. S. Goodman (Ed.) Change in Organizations, Jossey-Bass, San Francisco, 1982.

Whittington, R., A. Pettigrew, S. Peck, E. Fenton, and M. Conyon, "Change and Complementarities in the New Competitive Landscape: A European Panel Study, 1992-1996,” Organization Science, 10, (1999), 583-600.

Wilson, B. L. and H. D. Corbett, "Organization and Change: The Effects of School Linkages on the Quantity of Implementation,” Educational Administration Quarterly, 19, (1983), 85-104.

Womack, J. P., D. T. Jones, and D. Roos, The Machine that Changed the World, Rawson, New York, 1990. 


\section{Footnotes}

${ }^{1}$ Parallel to the literature on comparative statics, which is concerned with the optimal choices of a single decision maker, another stream of literature has concerned itself with the existence and the characterization of equilibrium outcomes of multi-player games in which players' payoff functions are supermodular, i.e., in which player $n$ 's payoff function is given by $f_{n}\left(\boldsymbol{x}_{n}, \boldsymbol{x}_{-n}, \boldsymbol{\theta}\right)$, with the condition that $f_{n}$ is supermodular in $\boldsymbol{x}_{n}$, and has increasing differences in $\boldsymbol{x}_{n}$ and $\boldsymbol{x}_{-n}$, and in $\boldsymbol{x}_{n}$ and $\theta$. Vives (1990) proves the existence of Nash equilibria in these types of games, while Milgrom and Roberts (1990b) show that smallest and largest pure Nash equilibria are nondecreasing functions of $\theta$, i.e., the bounds of the equilibrium set move monotonically with $\theta$. Similar to their extensions with respect to comparative statics results, Milgrom and Shannon (1994) loosen the assumptions of the game's structure to quasisupermodularity. Athey (forthcoming-b) further broadens the set-up, taking $\theta$ to be a vector of agents' types, which are private information of the agents, and derives conditions under which pure strategy Nash equilibria exist.

${ }^{2}$ The additive formulation has the appealing feature of not containing a "bliss point" with respect to $\alpha$. In other words, at no value of $\alpha$ does the effect of misperception vanish. In contrast, for the multiplicative case, if $\alpha=0$, misperceptions do not play a role. As a consequence, with multiplicative misperception, decision makers cannot misperceive independent activities—a somewhat uncomfortable assumption. Similarly, in the additive case, as the degree of interaction changes, the effective misperception is held constant, while in the multiplicative case, the effective misperception for a given $\delta$ changes as $\alpha$ changes.

${ }^{3}$ Similar functional forms arise in simple reliability models. Let $A$ and $B$ denote the probabilities that two components $a$ and $b$ will function. Then, if $a$ and $b$ work in series, the entire system will function with probability $A B$, whereas if $a$ and $b$ work in parallel, the system will function with probability $A+B-A B$. Thus, components working in series correspond to "complements" while components working in parallel correspond to substitutes. (I am grateful to Luis Garicano for pointing out this parallel.) 
${ }^{4}$ To avoid confusion resulting from possible relabeling of choice variables, activities are labeled such that their marginal benefit increases in $\alpha$. In the current set-up, this is equivalent to assuming that the two arguments of $V, A$ and $B$, are defined such that they are positive numbers, which is a natural restriction for investment- or effort levels.

${ }^{5}$ Also note that it is always beneficial to reduce misperceptions (see the proof for Proposition $1 \mathrm{~b}$ ). Thus, "signal" $z=\left(\alpha+\delta_{1}\right)$ is preferred to signal $z$ ' $=\left(\alpha+\delta_{2}\right)$ if $\left|\delta_{1}\right|<\left|\delta_{2}\right|$.

${ }^{6}$ Note the expectation is taken, in a sense, over a family of managers, each receiving a random draw $\delta_{i}$, and thus having the misperception $\left(\alpha+\delta_{i}\right)$. The interaction parameter $\alpha$ is still a fixed number. Section IV treats the more complex case in which $\alpha$ is also randomly distributed and the manager herself, when making her investment choices, is taking an expectation using the conditional distribution of $\alpha$ given her signal $z$.

${ }^{7}$ In solving for the Nash equilibrium it is assumed that managers' beliefs about the other manager's perceptions are correct, i.e., that M1 believes that M2 perceives the interaction to be $\left(\alpha+\delta_{2}\right)$ and that M2 believes that M1 perceives $\left(\alpha+\delta_{1}\right)$.

${ }^{8}$ The Milgrom and Roberts (1995) finding requires that the shocks are either equal or independently distributed. By using a quadratic functional form, Schaefer (1999) generalizes the finding to cases where shocks are more highly correlated on one side of the inequality than on the other. In particular, the functional form analyzed by Schaefer is given by: $V=\sum_{i=1}^{N-1} \sum_{j=i+1}^{N} \delta_{i j} x_{i} x_{j}$ with $\delta_{\mathrm{ij}} \geq 0$, guaranteeing that $V$ is supermodular in $\boldsymbol{x}$. 